\title{
On upstream influence in supersonic flows
}

\author{
By R. KRECHETNIKOV ${ }^{1}$ AND I. I. LIPATOV ${ }^{2}$ \\ ${ }^{1}$ California Institute of Technology, Pasadena, CA 91125, USA \\ ${ }^{2}$ Central Aero-Hydrodynamic Institute, Zhukovsky-3, 140160, Russia
}

(Received 1 October 2004 and in revised form 29 March 2005)

The general problem of propagation of three-dimensional disturbances in viscous supersonic flows is considered in the framework of characteristic analysis. Unlike previous results for linear disturbances we deduce a condition determining nonlinear characteristic surfaces which is exact and therefore allows both qualitative and quantitative studies of the speed of propagation as a function of various physical phenomena. These include negative and adverse pressure gradients, and effects of wall cooling and suction-blowing, which are studied in this work as an illustration of the general theory.

\section{Introduction}

Revival of interest in high-speed aerodynamics with the objective of developing technology ultimately applicable to vehicles from hypersonic aircraft to reusable space launchers (e.g. Hyper-X, NASA's multi-year hypersonic flight research program), necessitates consideration of remaining open questions. Among them is the wellknown problem of upstream propagation of disturbances in supersonic flows bounded by walls. While from inviscid theory it follows that an upstream influence occurs only in locally subsonic regions, Ferri (1939) experimentally found such an influence exerted by the boundary layer. This finding does not fit into the Prandtl hierarchical concept, in which the outer (external) flow is supersonic, and thus allows only downstream propagating disturbances in view of hyperbolicity, and the inner (internal) boundary layer flow is of parabolic type, and therefore also could not explain the upstream influence. This discrepancy generated the now well-known theory of viscous-inviscid interaction, which eventually explained boundary layer separation phenomena.

In his seminal work Lighthill (1953a) reviewed a large number of experimental and theoretical efforts to isolate two mechanisms by which the boundary layer transfers disturbances upstream. The first one, suggested by Oswatitsch \& Wieghardt (1941), amounts to a pressure gradient producing boundary layer thinning or thickening and thus inevitably changing the boundary layer shape (curvature) slightly upstream. This change, in turn, generates another pressure gradient, thus repeating the processes until disturbance decays far enough upstream. The second mechanism, introduced in works by Lees (1949) and Liepmann, Roshko \& Dhawan (1949), hinges on the existence of a sufficiently large compressive disturbance leading to separation of the boundary layer and thus resulting in modification of the external flow. As a consequence, the pressure rises ahead of the separation point and the boundary layer separates there too. While these two scenarios allowed classification of various experimental observations, they do not reveal a fundamental physical mechanism for their occurrence. However, from plain physical reasoning one might expect that the no-slip boundary condition at the wall inevitably introduces a subsonic sublayer inside the boundary layer, through the 

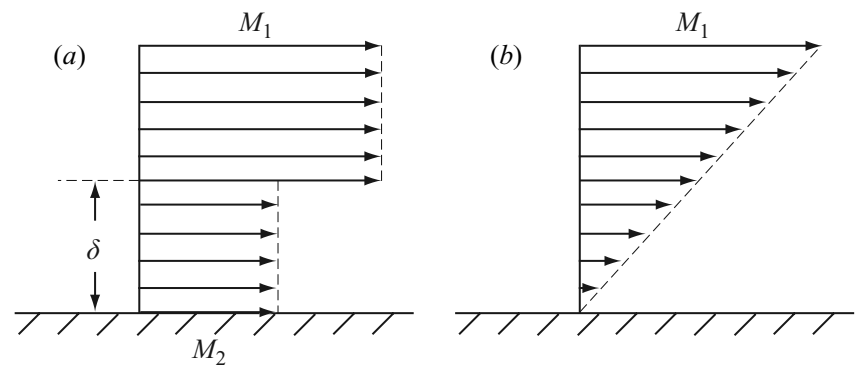

FIGURE 1. Previous approximate models: (a) Tsien et al. (1949), (b) Lighthill (1950),

FIGURE 1. Previous approximate models: (a) Tsien
(c) Lighthill (1953a).

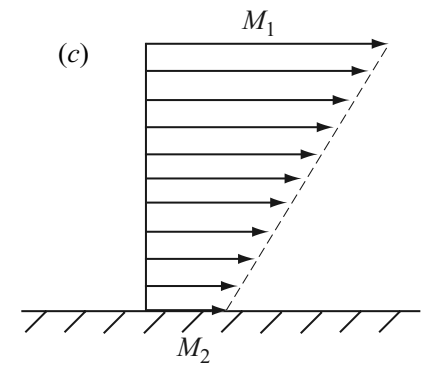

characteristic elliptic properties of which (steady subsonic flow obeys elliptic behaviour) the upstream influence can be transmitted, as conjectured by Howarth (1948).

One of the objectives of our work, in $\S 3$, is to introduce a rigorous derivation of this physical mechanism through the characteristic analysis inspired by the earlier works of Neiland (1974) and Lipatov (1996), which as indicated above originates in a two-component system - a parabolic boundary layer and a hyperbolic external flow through their interaction. Such a behaviour is now informally called weak ellipticity, and can be explained without going back to the complete Navier-Stokes description. While the conjecture of Howarth (1948) was distinguished by Lighthill (1953a) as a third independent mechanism of upstream influence, the subsonic sublayer determines a boundary layer response to pressure gradients and therefore plays a major role in the separation phenomena and in the origin of the first two mechanisms formulated above. This highlights the fundamental importance of understanding the processes of disturbance propagation. From the character of the two primary mechanisms of upstream influence, discussed above, it is obvious that propagation of disturbances of both small and large amplitudes is equally important. So far, only linear theories based on the idea of Howarth (1948) have been put forward to describe these processes. In particular, Tsien \& Finston (1949) replaced the flow structure by two layers of uniform flow - one with Mach number $M_{1}>1$ for the main stream and the other with $M_{2}<1$ for the boundary layer with subsonic width $\delta$ - as shown in figure $1(a)$. However, their inviscid theory does not determine the values of $M_{2}$ and $\delta$, upon which the attenuation of upstream influence depends. Another inviscid theory by Lighthill (1950) replaces the step-like structure on figure 1(a) by a continuous one, shown in figure $1(b)$. However, the lack of viscosity does not allow non-zero pressure gradients and therefore the distance of upstream influence turns out to be negligible, contradicting reality. This forced Lighthill (1953b) to postulate a non-zero Mach number at the wall as demonstrated in figure 1(c). The value of $M_{2}$ at the wall is determined from the solution in the inner viscous sublayer adjacent to the wall and described by incompressible equations since $M_{2}$ is assumed to be small.

As one can observe, all these works have an approximate nature which, even though they explain the basic physical mechanism, are not capable of giving a precise description. The latter is crucial if one wants to study, as in $\S 4$, the effect of the velocity profile shape on the propagation of disturbances or its dependence on various methods used in active boundary layer control, like temperature and suction-blowing at the wall. Besides the direction and the distance of disturbance propagation, the important characteristic of the process is the speed of propagation, since it defines the time scales of the separation phenomena, for example, relative to those of the main flow. 


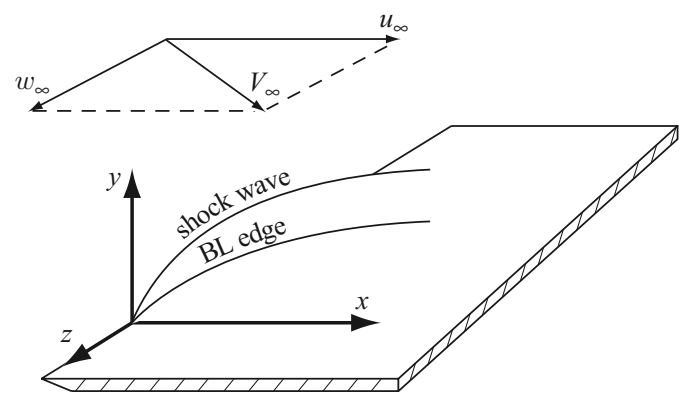

FiguRE 2. Flow geometry.

While Neiland (1970) demonstrated that perturbations propagate upstream over the whole length of the body in a hypersonic flow, there are no studies of the speed of propagation.

Lastly, as one can infer from the characteristic analysis, understanding zones of dependence and influence is important for consistent numerical formulations. The presence of upstream influence invalidates marching procedures commonly used in calculations of the boundary layer solutions as motivated by the parabolic properties of Prandtl's equations. The results obtained should be of help in formulating more advanced numerical techniques accounting for the viscous-inviscid interaction (cf. Veldman 1981).

\section{Problem formulation}

For definiteness we consider the flow of a perfect viscous gas over semi-infinite body with a sharp leading edge located at zero angle of attack, as shown in figure 2. Here we adopt the conventional boundary layer coordinates non-dimensionalized by a global streamwise length $l$ and a typical boundary layer thickness $\delta$ as follows: $\bar{x}=l x, \bar{y}=\delta y$, and $\bar{z}=l z$. The corresponding velocity components are scaled with respect to the free-stream $x$-component $u_{\infty}$ as $(\bar{u}, \bar{v}, \bar{w})=u_{\infty}\left(u, v_{r} v, w\right)$. Here the index $\infty$ stands for the free-stream quantity and the index $r$ for the order of magnitude of the non-dimensional quantity in the boundary layer, which may differ substantially from unity in view of drastic changes in the hydro- and thermodynamic quantities inside the boundary layer. For example, as follows from mass conservation, $v_{r}=\delta / l$, so that $v \sim O(1)$. Accordingly the time variable is defined as $\bar{t}=\left(l / u_{\infty}\right) t$, and the thermodynamic quantities are normalized in the same manner: density $\bar{\rho}=\rho_{\infty} \rho_{r} \rho$, pressure $\bar{p}=p_{\infty} p_{\underline{r}} p$, temperature $\bar{T}=T_{\infty} T_{r} T$, total enthalpy $\bar{H}=\left(u_{\infty}^{2} / 2\right) H$, viscosities $\bar{\mu}=\mu_{\infty} \mu_{r} \mu$ and $\bar{\lambda}=\mu_{\infty} \mu_{r} \lambda$. The physical system is characterized by a free-stream Reynolds number $R e_{\infty}$, the Reynolds number in the boundary layer $R e_{r}$, the Mach number $M_{\infty}$, and the Prandtl number $\mathrm{Pr}$, defined as

$$
R e_{\infty}=\frac{\rho_{\infty} u_{\infty} l}{\mu_{\infty}}, \quad R e_{r}=R e_{\infty} \frac{\rho_{r}}{\mu_{r}}, \quad M_{\infty}=\frac{u_{\infty}}{a_{\infty}}, \quad \operatorname{Pr}=\frac{\bar{\mu} \bar{C}_{p}}{\bar{\kappa}} .
$$

Here $a_{\infty}$ is a sound speed equal to $\left(\gamma R T_{\infty}\right)^{1 / 2}$ for perfect gas, $p=\rho T$, with specific heat ratio $\gamma$ and gas constant $R, \bar{C}_{p}$ the specific heat at constant pressure, and $\bar{\kappa}$ the heat conduction coefficient. The viscosity obeys a power-law dependence on temperature, $\mu=T^{\nu}$. In what follows, we consider the case $\operatorname{Pr}=1$ and disregard the effects of dissociation without limiting the generality of the theory. 
Substitution of these representations into the unsteady Navier-Stokes equations for compressible viscous gas and consideration of the non-degenerate limit $R e_{r} \sim l^{2} / \delta^{2} \gg 1$ corresponding to the boundary layer approximation with accuracy $O\left(R e_{r}^{-1}\right)$ yields

$$
\begin{aligned}
\mathrm{L} \rho & =-\rho \nabla \cdot \boldsymbol{v}, \\
\rho \mathrm{L} u & =-\Delta^{2} \frac{\partial p}{\partial x}+\frac{\partial}{\partial y}\left[\mu \frac{\partial u}{\partial y}\right], \\
\rho \mathrm{L} w & =-\Delta^{2} \frac{\partial p}{\partial z}+\frac{\partial}{\partial y}\left[\mu \frac{\partial w}{\partial y}\right], \\
\rho \mathrm{L} H & =2 \Delta^{2} \frac{\partial p}{\partial t}+\frac{\partial}{\partial y}\left[\mu \frac{\partial H}{\partial y}\right],
\end{aligned}
$$

where $\mathrm{L}=\partial_{t}+u \partial_{x}+v \partial_{y}+w \partial_{z}$ is a convection operator, $\Delta^{2}=T_{r} / \gamma M_{\infty}^{2}$, and the pressure is independent of the thin dimension $y$, i.e. $p=p(t, x, z)$ to leading order with accuracy $O\left(R e_{r}^{-1}\right)$. We work sufficiently far from the leading edge that the shock wave and the boundary layer are separated by an inviscid region, as shown in figure 2 . This remains valid not only for supersonic flows, but also for hypersonic ones, $M_{\infty} \gg 1$, if the hypersonic interaction parameter $\chi_{\infty}=M_{\infty}^{\nu+2} R e_{\infty}^{-1 / 2}=o\left(M_{\infty}^{2}\right)$, since as one can easily demonstrate the ratio of mass fluxes through the compressed layer and boundary layer is $\Psi_{c l} / \Psi_{\delta} \sim M_{\infty}^{2} / \chi_{\infty}$ (cf. Hayes \& Probstein 1959). System (2.1) is subject to the usual boundary conditions,

$$
\begin{array}{ccc}
y=0: & u=0, \quad v=v_{w}, \quad w=0, \quad H=H_{w} ; \\
y=\infty: \quad u=1, \quad w=w_{e}, \quad H=H_{e},
\end{array}
$$

where $v_{w}$ and $H_{w}$ stand for the mass and temperature control at the wall, and $H_{e} \rightarrow$ $1+w_{e}^{2}$ in the hypersonic case. The last boundary condition, accounting for the interaction between the boundary layer and outer flow, is modelled here with the local tangent-wedge formula (i.e. Newton's formula; cf. Hayes \& Probstein 1959), according to which the pressure on the body elementary area depends unsteadily only on its orientation in space relative to the incoming flow and is independent of the shape of the rest of the body,

$\frac{1}{\sqrt{1+w_{e}^{2}}}\left(\frac{\partial \delta^{*}}{\partial t}+\frac{\partial \delta^{*}}{\partial x}+w_{e} \frac{\partial \delta^{*}}{\partial z}\right)=f(p)=\frac{p p_{r}-1}{M_{\infty}}\left[\gamma^{2}+\frac{\gamma(\gamma+1)}{2}\left(p p_{r}-1\right)\right]^{-1 / 2}$.

This condition, readily obtainable from the unsteady relations at the shock wave for a perfect gas, is applicable to supersonic as well as to hypersonic flows, including both regimes of interaction - strong, when $\delta^{*} \sim x^{3 / 4}$, and weak, $\delta^{*} \sim x^{1 / 2}$, as in the classical boundary layer (cf. Hayes \& Probstein 1959).

To simplify the subsequent analysis we perform a standard transformation of system (2.1) to Dorodnitsyn variables generalized to the unsteady case (cf. Stewartson 1965),

$$
(t, x, y, z) \rightarrow\left(\tau=t, \xi=x, \eta=\int_{0}^{y} \rho \mathrm{d} \widehat{y}, \zeta=z\right),
$$

so that system (2.1) assumes the form

$$
\begin{aligned}
0 & =\frac{\partial u}{\partial \xi}+\frac{\partial \widetilde{v}}{\partial \eta}+\frac{\partial w}{\partial \zeta}, \\
\mathrm{L} u & =-\frac{\Delta^{2}}{\rho} \frac{\partial p}{\partial \xi}+\frac{\partial}{\partial \eta}\left[\rho \mu \frac{\partial u}{\partial \eta}\right],
\end{aligned}
$$




$$
\begin{aligned}
& \mathrm{L} w=-\frac{\Delta^{2}}{\rho} \frac{\partial p}{\partial \zeta}+\frac{\partial}{\partial \eta}\left[\rho \mu \frac{\partial w}{\partial \eta}\right], \\
& \mathrm{L} H=2 \Delta^{2} \frac{\partial p}{\partial \tau}+\frac{\partial}{\partial \eta}\left[\rho \mu \frac{\partial H}{\partial \eta}\right],
\end{aligned}
$$

where $\widetilde{v}=\eta_{t}+u \eta_{x}+w \eta_{z}+\rho v$ and the convective operator $\mathrm{L}$ is the same as before, except for the substitution $v \rightarrow \widetilde{v}$. Also, for simplicity of notation, we introduce two stream functions $\psi$ and $\phi$ defined according to $u=\psi_{\eta}, \widetilde{v}=-\psi_{\xi}-\phi_{\zeta}, w=\phi_{\eta}$.

Lastly, the boundary layer thickness appearing in the interaction condition (2.2) can be expressed in generic form as an integral,

$$
\delta^{*}=\int_{0}^{+\infty} f(\boldsymbol{v}, \rho, H) \mathrm{d} y=\int_{0}^{+\infty} \frac{f(\boldsymbol{v}, \rho, H)}{\rho} \mathrm{d} \eta,
$$

where the form of $f(\boldsymbol{v}, \rho, H)$ in general depends on the definition of $\delta^{*}$ (i.e. velocity, displacement, momentum thicknesses; cf. Schlichting 1962). While in the supersonic case this choice influences somewhat the final relation for the characteristics, in the hypersonic case this variation disappears since the boundary layer outer edge is very distinct in view of the substantial difference in densities inside and outside the boundary layer, as visually seen in experiments. In the latter case, $f(\boldsymbol{v}, \rho, H)=1+O\left(\chi_{\infty} /\right.$ $\left.M_{\infty}^{2}\right)$ for strong interaction and $f(\boldsymbol{v}, \rho, H)=1+O\left(M_{\infty}^{-2}\right)$ for weak interaction, so that the final form is

$$
\delta^{*}=\frac{\gamma-1}{2 \gamma \Delta^{2}} \frac{I}{p}, \quad I \equiv \int_{0}^{+\infty}\left[H-u^{2}-w^{2}\right] \mathrm{d} \eta,
$$

where the definition of total enthalpy was used.

\section{Theory: characteristic analysis}

It is known that there are many ways to introduce the concept of characteristics without changing its meaning. In our characteristic analysis we essentially follow the way in which Petrovsky (1954) defined the characteristic surface in proving the Cauchy-Kovalevskaya theorem: an initial value problem has no unique solution (i.e. the Cauchy problem is ill-posed) if the initial data are posed on a characteristic surface. This allows a straightforward determination of this surface $\Omega(\boldsymbol{x})$ by making a transformation from original independent variables $\boldsymbol{x}$ to new ones $\boldsymbol{\Omega}(\boldsymbol{x})$ in which resolution for the highest derivative of the solution becomes impossible. While the characteristics are the integral part of the problem (even if it is in a constant state), they are the paths along which linear and nonlinear, continuous and discontinuous disturbances propagate. Therefore, the characteristic analysis allows considerably more general results to be obtained compared to the linear ones based on ad hoc velocity profiles by Lighthill (1953b).

In view of the thin layer approximation in (2.1), we are interested in the propagation of disturbances only in the $(\xi, \zeta)$-plane, i.e. parallel to the body surface. In this respect the unsteady characteristic surfaces associated with this plane are exact. However, as the analysis indicates they are equivalent to subcharacteristics, a concept introduced in the singular perturbation methods (cf. Cole 1968) as characteristics associated with the lower derivatives in partial differential equations. While the role played by subcharacteristics in these methods is secondary, the works by Wang $(1971,1975)$ on three-dimensional incompressible boundary layers revealed their major role in determining zones of influence and dependence, and the structure of solution. 


\subsection{Hypersonic boundary layer}

In the light of the above we associate the propagating quantity (initial data) with the characteristic surface $\Omega$, which in view of the thin layer approximation depends only on time-like variables, that is $\Omega=\Omega(\tau, \xi, \zeta)$, so that the disturbances propagate with the same speed across the boundary layer thin dimension $y$. Therefore, the transformation assumes the form $(\tau, \xi, \eta, \zeta) \rightarrow(\Omega(\tau, \xi, \zeta), \eta)$, which when applied to (2.4), (2.2), (2.6) yields a system for $\delta_{\Omega}^{*}, p_{\Omega}, \psi_{\Omega}, \phi_{\Omega}, H_{\Omega}$, which after elementary elimination of $\delta_{\Omega}^{*}$ becomes

$$
\begin{aligned}
f(p) & =\frac{\widetilde{\Delta}_{e}}{\sqrt{1+w_{e}^{2}}} \frac{1}{p^{2}}\left[p I_{\Omega}-I p_{\Omega}\right], \\
\widetilde{\Delta} \psi_{\eta \Omega}-D \psi_{\eta \eta} & =-\frac{\gamma-1}{2 \rho \gamma} \Omega_{\xi} p_{\Omega}+C_{u}, \\
\widetilde{\Delta} \phi_{\eta \Omega}-D \phi_{\eta \eta} & =-\frac{\gamma-1}{2 \rho \gamma} \Omega_{\zeta} p_{\Omega}+C_{w}, \\
\widetilde{\Delta} H_{\Omega}-D H_{\eta} & =-\frac{\gamma-1}{\rho \gamma} \Omega_{\tau} p_{\Omega}+C_{H},
\end{aligned}
$$

where $D=\Omega_{\xi} \psi_{\Omega}+\Omega_{\zeta} \phi_{\Omega}, \widetilde{\Delta}=\Omega_{\tau}+\psi_{\eta} \Omega_{\xi}+\phi_{\eta} \Omega_{\zeta}, \widetilde{\Delta}_{e}=\left.\widetilde{\Delta}\right|_{\eta=+\infty}$, and $C_{f}=\left[\rho \mu f_{\eta}\right]_{\eta}$. Recalling the definition of the integral $I$ from (2.6), one can observe that on eliminating $\psi_{\Omega}, \phi_{\Omega}, H_{\Omega}$ from $I_{\Omega}=\int_{0}^{+\infty}\left[H_{\Omega}-2 \psi_{\eta} \psi_{\eta \Omega}-2 \phi_{\eta} \phi_{\eta \Omega}\right] \mathrm{d} \eta$ one should arrive at only one equation for pressure. Multiplying equation (3.1b) by $\Omega_{\xi}$ and equation (3.1c) by $\Omega_{\zeta}$ and adding the results, one obtains

$$
D=\widetilde{\Delta} \int_{0}^{\eta} \frac{\Omega_{\xi} C_{u}+\Omega_{\zeta} C_{w}-\left(\Omega_{\xi}^{2}+\Omega_{\zeta}^{2}\right)((\gamma-1) / 2 \rho \gamma) p_{\Omega}}{\widetilde{\Delta}^{2}} \mathrm{~d} \eta .
$$

Using this expression one finds $\psi_{\eta \Omega}, \phi_{\eta \Omega}, H_{\Omega}$ from equations (3.1b-d), and substituting the results into $(3.1 a)$ gives the final equation for the pressure on a characteristic surface:

$$
N \frac{\mathrm{d} p}{\mathrm{~d} \Omega}=\gamma p\left\{\frac{\sqrt{1+w_{e}^{2}}}{\widetilde{\Delta}_{e}} p f(p)-\widetilde{f}\right\} .
$$

Since $\widetilde{f}$ is not a function of pressure and its derivative(s), the characteristic equation is defined by the vanishing of the factor $N$ in the above equation, i.e.

$$
N=\frac{\gamma-1}{2} \int_{0}^{+\infty} \frac{\left(H-u^{2}-w^{2}\right)^{2}}{[a-u \cos (\omega)-w \sin (\omega)]^{2}} \mathrm{~d} \eta-\int_{0}^{+\infty}\left(H-u^{2}-w^{2}\right) \mathrm{d} \eta=0 .
$$

Here we have introduced the speed of disturbances propagation $a$ and the angle $\omega$ between $x$-axis and direction of propagation, defined as

$$
a=\Omega_{\tau}\left[\Omega_{\xi}^{2}+\Omega_{\zeta}^{2}\right]^{-1 / 2}, \quad a \cos \omega=-\frac{\Omega_{\tau} \Omega_{\xi}}{\Omega_{\xi}^{2}+\Omega_{\zeta}^{2}}, \quad a \sin \omega=-\frac{\Omega_{\tau} \Omega_{\zeta}}{\Omega_{\xi}^{2}+\Omega_{\zeta}^{2}} .
$$

The characteristic condition (3.3) allows one to determine the speed of propagation depending upon the angle in the $(\xi, \zeta)$-plane for given profiles of velocity and total enthalpy. This will be the subject of $\S 4$.

Some physical insights into the meaning of (3.3) can be attained by writing this condition in the steady case, $a=0$, as applied to the cylindrical ejector supersonic propelling nozzle (refer to figure 3) with constant Mach numbers $M_{j}$ and $M_{s}$ in the main 


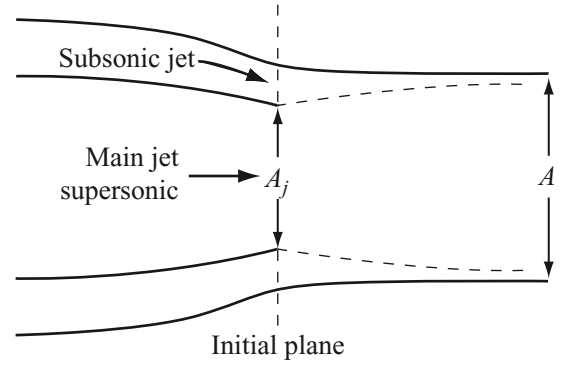

FiguRE 3. Ejector nozzle.

and secondary jets respectively. As a result, we recover the condition of Pearson, Holliday \& Smith (1958),

$$
A_{j}\left(1-M_{j}^{-2}\right)+A_{s}\left(1-M_{s}^{-2}\right)=0, A_{j}+A_{s}=A=\mathrm{const},
$$

illustrating the evolution of the jet after leaving the initial plane: thickening of the supersonic area $A_{j}$ reduces the subsonic one $A_{s}$. Thus one can anticipate that this 'conservation' property - balance of sub- and supersonic parts of the velocity profile - will determine the speed of propagation in the unsteady case.

It is also notable that equation (3.3) has some similarities - an analogous singular denominator $(u-a)^{2}$ under the integral sign - with the long wave study by Teshukov (1994) in a vortex barothropic fluid under the shallow water approximation. Despite the lack of viscosity in the latter problem, it has some common features with ours, namely the presence of vorticity, nonlinear convective operator, thin layer approximation and integro-differential character of the problem. The analogue of interaction in the problem considered by Teshukov (1994) comes from the $y$-momentum equation, $p_{y}=-\rho$, accounting for the barotropic behaviour.

\subsection{Generalization to the supersonic boundary layer}

The finite Mach number case can be treated essentially as in the previous subsection with the only complication coming from the absence of a distinct boundary layer outer edge as opposed to the hypersonic case. This makes the function $f$, defined in (2.5), depend on $(\boldsymbol{v}, \rho, H)$ as opposed to $f \simeq 1$ in the hypersonic case. Here, as an illustration, we consider the case of velocity thickness, so that $f=1-u$ for the two-dimensional boundary layer. Following the procedure outlined in the previous subsection yields an equation for pressure analogous to (3.2), so that the characteristic equation is given by

$$
\begin{aligned}
N= & \frac{\gamma-1}{2} \int_{0}^{+\infty} \frac{(1-u)\left(H-u^{2}\right)^{2}}{(u-a)^{2}} \mathrm{~d} \eta-\int_{0}^{+\infty}(1-u)\left(H-u^{2}\right) \mathrm{d} \eta \\
& +\frac{\gamma-1}{2} \int_{0}^{+\infty}\left(H-u^{2}\right)\left\{\frac{1}{(u-a)^{2}} \int_{0}^{\eta}\left(H-u^{2}\right) \mathrm{d} \widehat{\eta}-u_{\eta} \int_{0}^{\eta} \frac{H-u^{2}}{(u-a)^{2}} \mathrm{~d} \widehat{\eta}-\frac{H-u^{2}}{u-a}\right\} \mathrm{d} \eta
\end{aligned}
$$

In the hypersonic limit the entire second line of this expression vanishes while the first line naturally converges to (3.3) with $\omega=0$. Solving (3.4) is no more complicated than solving (3.3) for the disturbance speed $a$ for known velocity and enthalpy profiles.

\subsection{Generalization to the flow in a corner}

The same type of analysis can be applied not only to flows over smooth flat surfaces but also to flows in corners, where strong upstream influence was observed 


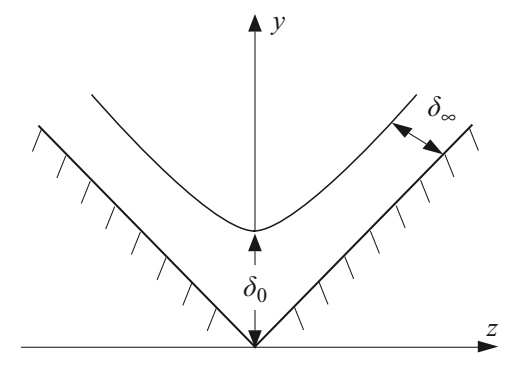

FIGURE 4. Flow in a corner.

experimentally (V. N. Ostras, private communication). As an example we consider the viscous flow of a perfect gas in a corner as shown in figure 4; the free-stream velocity is directed along the $x$-axis. The boundary layer formulation (2.1) is clearly not applicable anymore, and for a self-consistent formulation one needs to take into account the first two terms in the asymptotic expansion for the pressure, $p=p_{0}(t, x)+$ $\epsilon p_{1}(t, x, y, z)+\ldots$, where $\epsilon=\left[\Delta R e_{r}\right]^{-2} \ll 1$. This produces a set of parabolized Navier-Stokes equations (for $\operatorname{Pr}=1$ ),

$$
\begin{aligned}
\mathrm{L} \rho & =-\rho \operatorname{div} \boldsymbol{v}, \\
\rho \mathrm{L} u & =-\Delta^{2} \frac{\partial p_{0}}{\partial x}+\frac{\partial}{\partial y}\left[\mu \frac{\partial u}{\partial y}\right]+\frac{\partial}{\partial z}\left[\mu \frac{\partial u}{\partial z}\right], \\
\rho \mathrm{L} v & =-\Delta^{2} \frac{\partial p_{1}}{\partial z}+\frac{\partial}{\partial x}\left[\mu \frac{\partial u}{\partial y}\right]+\frac{\partial}{\partial y}\left[2 \mu \frac{\partial v}{\partial y}+\lambda \nabla \cdot v\right]+\frac{\partial}{\partial z}\left[\left(\frac{\partial v}{\partial z}+\frac{\partial w}{\partial y}\right)\right], \\
\rho \mathrm{L} w & =-\Delta^{2} \frac{\partial p_{1}}{\partial z}+\frac{\partial}{\partial x}\left[\mu \frac{\partial u}{\partial z}\right]+\frac{\partial}{\partial y}\left[\mu\left(\frac{\partial v}{\partial z}+\frac{\partial w}{\partial y}\right)\right]+\frac{\partial}{\partial z}\left[2 \mu \frac{\partial w}{\partial z}+\lambda \nabla \cdot v\right], \\
\rho \mathrm{L} H & =2 \Delta^{2} \frac{\partial p_{0}}{\partial t}+\frac{\partial}{\partial y}\left[\mu \frac{\partial H}{\partial y}\right]+\frac{\partial}{\partial z}\left[\mu \frac{\partial H}{\partial z}\right] .
\end{aligned}
$$

In this physical set-up we are interested in understanding the propagation of disturbances along the corner edge, which is best described by considering the problem in a neighbourhood of the plane of symmetry, so that the interaction condition is given by a simplified version of (2.2):

$$
f(p)=\frac{\partial \delta^{*}}{\partial t}+\frac{\partial \delta^{*}}{\partial x}, \quad \delta^{*}=\delta_{0}+\frac{z^{2}}{2\left(\delta_{0}-\sqrt{2} \delta_{\infty}(x)\right)}+\ldots,
$$

where $\delta_{0}$ is a boundary layer thickness in the plane of symmetry and $\delta_{\infty}$ is a thickness at infinity, as illustrated in figure 4.

In this formulation the disturbance evolution is governed by the $x$-momentum and energy equations: thus after transformation to Dorodnitsyn and characteristic variables, $(\tau, \xi, \eta, \zeta) \rightarrow(\Omega(\tau, \xi), \eta, \zeta)$, one obtains a two-dimensional version of (3.3) with $\omega=0$ and $w \equiv 0$, which allows one to determine the speed of propagation in a neighbourhood of the symmetry plane.

\section{Application: a hypersonic yawed wing}

The use of the characteristic equation (3.3) for computing disturbance propagation is especially straightforward in the case of small-amplitude disturbances. In this case, the profiles of velocity and enthalpy correspond to the basic state (steady or unsteady). 


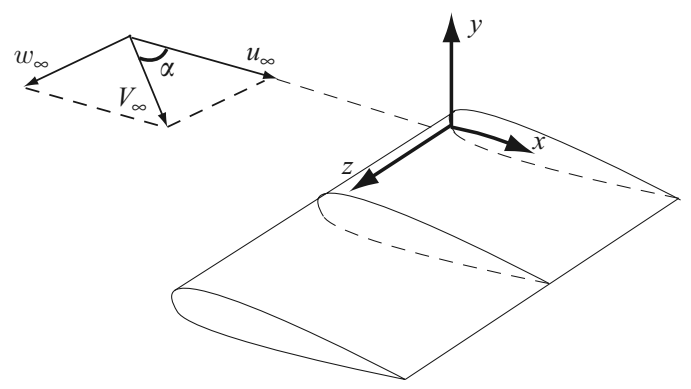

FiguRE 5. Yawed wing.

As an example of an application, cf. figure 5 and Schlichting (1962), we consider the case of a steady hypersonic flow over a yawed wing of infinite span at zero lift (zero angle of attack), which is suited to illustrational purposes. In this case the potential flow depends on $x$ only, so that system (2.4) is substantially simplified in view of the lack of dependence on the $z$-coordinate. Taking into account this fact and applying further transformation to the boundary layer equations in Dorodnitsyn variables (2.4), $\xi \rightarrow \widetilde{\xi}=\int_{0}^{\xi} \rho_{w} \mu_{w} u_{\delta} \mathrm{d} \xi, \eta \rightarrow \widetilde{\eta}=\frac{u_{\delta}}{f(\xi)} \eta$, and $\psi=f(\xi) \Psi(\widetilde{\eta}), \phi=f(\xi) \Phi(\widetilde{\eta}), H=H_{\delta} g$, where $f(\xi)=(2 \widetilde{\xi})^{1 / 2}$ and $H_{\delta}=u_{\delta}^{2}$, for the case of linear dependence of viscosity on temperature, $\mu=T$, and a power-law pressure distribution, $p=\xi^{n}$, we arrive at a system of Falkner-Skan type:

$$
\left.\begin{array}{r}
\Psi_{\tilde{\eta} \tilde{\eta} \tilde{\eta}}+\Psi \Psi_{\tilde{\eta} \tilde{\eta}}+\beta\left(g-\Psi_{\tilde{\eta}}^{2}-\Phi_{\tilde{\eta}}^{2}\right)=0 \\
\Phi_{\tilde{\eta} \tilde{\eta} \tilde{\eta}}+\Psi \Phi_{\tilde{\eta} \tilde{\eta}}=0 \\
g_{\tilde{\eta} \tilde{\eta}}+\Psi g_{\tilde{\eta}}=0
\end{array}\right\}
$$

with the boundary conditions

$$
\begin{aligned}
& \tilde{\eta}=0: \quad \Psi=\Psi_{w}, \quad \Psi_{\tilde{\eta}}=\Phi_{\tilde{\eta}}=0, \quad g=g_{w}, \\
& \widetilde{\eta}=\infty: \quad \Psi_{\tilde{\eta}}=1, \quad \Phi_{\tilde{\eta}}=\tan \alpha, \quad g=\cos ^{-2} \alpha .
\end{aligned}
$$

Here $\alpha$ is an angle between the vector of free-stream velocity and the $x$-axis, and

$$
\beta=-\frac{\gamma-1}{\gamma} \frac{n}{n+1}
$$

is the classical Falkner-Skan constant.

The solution of (4.1) was constructed for two types of flow: one corresponding to an induced pressure distribution with $n=-\frac{1}{2}$, and the other corresponding to an adverse pressure gradient with $n>0$ (the results in figure $6(b)$ are shown for $n=0.1$ ). System (4.1) was integrated by a standard finite-difference method with care paid to non-uniqueness of the solution for $n>0$ (equivalent to negative $\beta$, known since the work of Stewartson 1954). In the case $n>0$ we were interested in the branch of the solution corresponding to reverse flow (negative friction at the wall).

\subsection{Directional diagram}

The self-similar solution obtained by integration of (4.1) refers to the flow on a yawed wing of infinite span. This solution can be used in the characteristic equation (3.3) to determine the vector $\left(a_{\xi}, a_{\zeta}\right)$, which forms the directional diagram common in acoustics. 

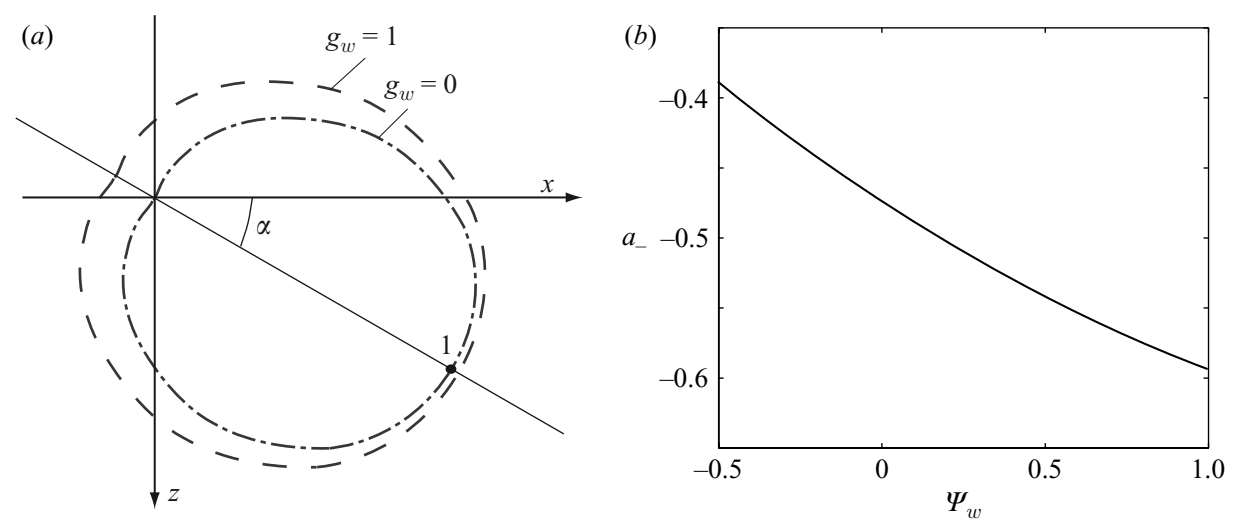

FIgURE 6. Functionality of propagation speed. (a) Directional diagram; $\Psi_{w}=0$.

(b) $a_{-}\left(\Psi_{w}\right)$ in reverse flow; $g_{w}=1$.
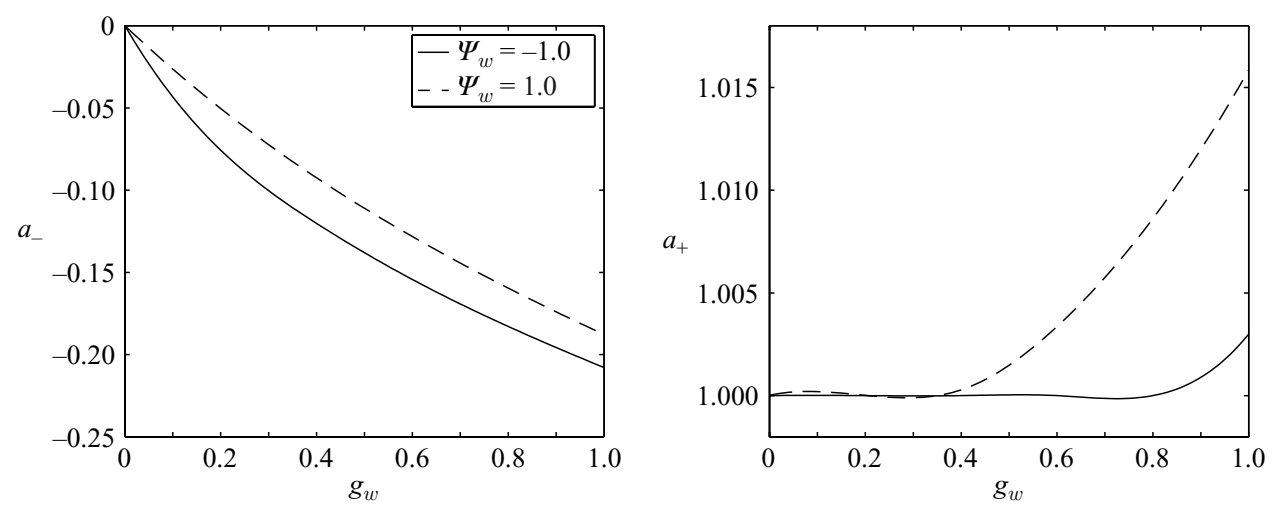

FIGURE 7. Influence of the wall temperature, $g_{w}$, and mass flux, $\Psi_{w}$. (a) Upstream speed $a_{-}$. (b) Downstream speed $a_{+}$.

This diagram, computed for angle $\alpha=30^{\circ}$ and shown in figure 6(a), illustrates how the disturbance would propagate with time after introduction at some point in the boundary layer, e.g. by an incident shock wave. The influence of wall cooling, $g_{w}=0$, is in the reduction of the speed of propagation in all directions relative to the normal temperature, $g_{w}=1$, and at $g_{w}=0$ the upstream influence disappears completely the phenomenon to be discussed in the next subsection.

\subsection{Influence of active control}

The upstream and downstream influences were studied under the effects of wall temperature and mass flux through the wall. The range of corresponding parameters considered here was $g_{w} \in[0,1]$ - from perfectly cold to normal temperature wall and $\Psi_{w} \in[-1,1]$, with negative values corresponding to suction, and positive ones to blowing.

Figures 7(a) and 7(b) demonstrate the dependence of the upstream $a_{-}$and downstream $a_{+}$speeds respectively on the temperature factor $g_{w}$ for two limiting values of mass flux $\Psi_{w}= \pm 1$. One can observe that an increase in suction reduces $a_{-}$due to a reduction of the subsonic part of the velocity profile. Wall cooling also leads to thinning of the subsonic part, including the elimination of upstream propagation at a perfectly cold wall, thus confirming the conclusions of the triple-deck analysis by 
Brown, Cheng \& Lee (1990), who showed that lowering the wall temperature reduces the triple-deck dimension and hence the degree of upstream influence. Figure $6(b)$ illustrates the influence of an adverse pressure gradient, $p \sim \xi^{0.1}$, on the upstream propagation: the presence of reversed flow in the wall vicinity provides an additional convective mechanism to convey disturbances and significantly amplifies the speed of propagation.

\section{Conclusions}

In this work we have put the description of upstream influence in supersonic flow on a systematic and rigorous footing. Unlike the previous approximate linear modelling by Lighthill (1953b), our analysis yields an exact relation from which one can determine both linear and nonlinear disturbance characteristics. The characteristic equation was derived for three-dimensional hypersonic boundary layers interacting with inviscid flow, and was generalized to supersonic boundary layer flow and flow in a corner, described by the parabolized Navier-Stokes equations. As an illustration we considered three-dimensional flow over a yawed wing, and studied the disturbance propagation characteristics as functions of the direction (relative to the free stream velocity vector), pressure gradient (positive and negative), and active control variables - temperature and mass flux at the wall.

\section{REFERENCES}

Brown, S. N., Cheng, H. K. \& LeE, C. J. 1990 Inviscid-viscous interaction on triple-deck scales in a hypersonic flow with strong wall cooling. J. Fluid Mech. 220, 309-337.

Cole, J. D. 1968 Perturbation Methods in Applied Mathematics. Blaisdell.

FERrI, A. 1939 Atti di guidonia. Tech. Rep. 17. Engl. transl. Experimental results with airfoils tested in the high-speed tunnel at Guidonia. NACA TM 946 (1940).

Hayes, W. D. \& Probstein, R. F. 1959 Hypersonic Flow Theory. Academic Press.

HowARTH, L. 1948 The propagation of steady disturbances in a supersonic stream bounded on one side by a parallel subsonic stream. Proc. Camb. Phil. Soc. 44, 380-390.

LEES, L. 1949 Interaction between the laminar boundary layer over a plane surface and an incident oblique shock wave. Aero. Engng lab. Tech. Rep. 143 Princeton.

Liepmann, H. W., Roshko, A. \& Dhawan, S. 1949 On the reflection of shock waves from boundary layers. Tech. Rep. Galcit, Caltech.

Lighthill, M. J. 1950 Reflection at a laminar boundary layer of a weak steady disturbance to a supersonic stream, neglecting viscosity and heat conduction. Q. J. Mech. 3, 303-325.

Lighthill, M. J. 1953a On boundary layers and upstream influence. I. A comparison between subsonic and supersonic flows. Proc. R. Soc. Lond. A 217, 344-357.

Lighthill, M. J. $1953 b$ On boundary layers and upstream influence. II. Supersonic flows without separation. Proc. R. Soc. Lond. A 217, 478-507.

Lipatov, I. I. 1996 The propagation of perturbations in supersonic boundary layers. J. Appl. Math. Mech. 60, 455-462.

NeILAND, V. Y. 1970 Upstream propagation of disturbances under condition of interation of hypersonic flow with boundary layer. Izv. Akad. Nauk., Mekh. Zhid. Gaz. 4, 40-49.

NeILAND, V. Y. 1974 On the theory of interaction between a hypersonic flow and a boundary layer for separated two-dimensional and three-dimensional flows. Uch. Z. TsAGI 5, 70-79.

Oswatitsch, K. \& Wieghardt, K. 1941 German wartime report. Tech. Rep. Reprinted as Tech. Mem. Natl Adv. Comm. Aero. Wash. 1189 (1948).

Pearson, H., Holliday, J. B. \& Smith, S. F. 1958 A theory of the cylindrical ejector supersonic propelling nozzle. J. R. Aeronaut. Soc. 62, 746-751.

Petrovsky, I. G. 1954 Partial Differential Equations. Interscience.

Schlichting, H. 1962 Boundary Layer Theory. McGraw-Hill. 
Stewartson, K. 1954 Further solutions of the Falkner-Skan equation. Proc. Camb. Phil. Soc. 50, $454-465$.

Stewartson, K. 1965 The Theory of Laminar Boundary Layers in Compressible Fluids. Oxford University Press.

Teshukov, V. M. 1994 Long waves in a vortex barothropic fluid. J. Appl. Mech. Tech. Phys. 35, $17-26$.

Tsien, H. S. \& Finston, M. 1949 Interaction between parallel streams of subsonic and supersonic velocities. J. Aero. Sci. 16, 515-528.

Veldman, A. E. P. 1981 New, quasi-simultaneous method to calculate interacting boundary layers. AIAA J. 19, 79-85.

WANG, K. C. 1971 On the determination of the zones of influence and dependence for threedimensional boundary-layer equations. J. Fluid Mech. 48, 397-404.

WANG, K. C. 1975 Aspects of "multitime initial-value problem" originating from boundary layer equations. Phys. Fluids 18, 951-955. 